\title{
A propos d'un vol nuptial de Leptoconops (L.) bezzii Noé, 1905
}

\author{
par Jean-A. RIOUX et Suzanne DESCOUS
}

A lire attentivement les descriptions princeps de G. Noé (1905), les observations de J. J. Kieffer (1921) et H. F. Carter (1921) plusieurs critères morphologiques permettent de distinguer les femelles de Leptoconops bezzii et de Leptoconops irritans. Citons en particulier la plus grande longueur des palpes, la présence d'une véritable dent tarsale subapicale et l'absence de spermathèque rudimentaire chez Leptoconops bezzii.

Malheureusement, si le mâle de cette dernière espèce a pu être assez souvent capturé, celui de Leptoconops irritans reste inconnu jusqu'à ce jour. Bien plus, la plupart des auteurs (M. Bezzi, sine dat., H. Harant et D. Brunel, 1950) ont récolté ensemble les deux espèces sous la forme femelle pour Leptoconops irritans et mâle pour Leptoconops bezzii. Nous-même, dans un précédent travail (1965), avons pu déterminer dans un lot de Leptoconops récolté en Roussillon par M. Lamontellerie, le curieux mélange Leptoconops bezzii mâle et Leptoconops irritans femelle.

Dans la présente note, nous voudrions apporter une nouvelle contribution à la systématique de ces deux espèces en faisant état d'une observation de valeur, réalisée en Camargue le 3 juin 1966.

Ce jour, à l'entrée de la Réserve biologique de Camargue, au lieudit * les Salins de Badon », sur la route D. 36, nous observons un vol nuptial de Leptoconops (température $27^{\circ} 5$, humidité $\left.40 \%\right)$. Malgré un vent assez fort $(2 \mathrm{~m} / \mathrm{s})$, ce vol persiste pendant plusieurs heures. Tantôt dispersé, tantôt regroupé entre les rafales, il évolue entre un et trois mètres au-dessus du sol.

Nos captures, effectuées au filet humide, sont immédiatement plongées dans l'alcool à $70^{\circ}$.

Après dépouillement et examen, nous déterminons 320 Leptoconops bezzii mâles et 6 Leptoconops irritans femelles. Aucune femelle répondant à la description princeps de Leptoconops bezzii n'est identifiée. En particulier, les six exemplaires femelles possèdent une spermathèque rudimentaire bien visible; les palpes sont longs et les griffes tarsales sont dépourvues de dent subapicale. 
Au demeurant, si l'observation privilégiée de ce vol nuptial apporte un argument supplémentaire en faveur de l'identité systématique Leptoconops bezzii ó-Leptoconops irritans + , d'autres données sont encore nécessaires pour asseoir définitivement la synonymie. En cette matière, on doit attendre beaucoup de l'étude biométrique des deux espèces ainsi que de la détection des biotopes larvaires correspondants.

\section{Bibliographie}

Carter (H. F.), 1921. - A revision of the genus Leptoconops Skuse. Bull. of Entomol. Res., $12,28 \mathrm{p}$.

Harant (H.) et Brunel (D.), 1950. - Sur les Leptoconops s. lato du Midi Méditerranéen. Vie et Milieu, 240-242.

KiefFer (J. J.), 1921. - Nouvelles observations sur les Diptères piqueurs de la tribu des Cératopogoninae. Arch. Inst. Pasteur Afr. Nord, 1, p. 226.

NoÉ (G.), 1905. - Un nuove genere appartenente alla famiglia Chironomidae. Atti R. Accad. Lincei, série 5, 14, 114-120.

Rioux (J. A.) et Descous (S.), 1965. - Présence en France de Leptoconops (Leptoconops) bezzii Noé, 1905, nouvelle mention de Leptoconops (Leptoconops) irritans Noé, 1905. Feuille des Naturalistes.

(Laboratoire d'Ecologie médicale et de Pathologie parasitaire, Faculté de Médecine, F. 34 -Montpellier) 